\title{
Pollen-Allergie
}

\section{Sind Lipide das Zünglein an der Waage?}

\author{
Nach bisheriger Lehrmeinung setzen Pollen Allergene frei, die \\ über die Haut oder Schleimhaut in den Körper eindringen und hier \\ eine spezifische allergische Reaktion hervorrufen können. Bislang \\ war jedoch unklar, mit welchem Mechanismus exogene Allergene \\ die für Allergien typische Verschiebung in Richtung Th2-Antwort \\ auslösen. Die Arbeitsgruppe um Prof. Traidl-Hoffmann hat die Pollen \\ daher näher untersucht und eine mögliche Erklärung gefunden.
}

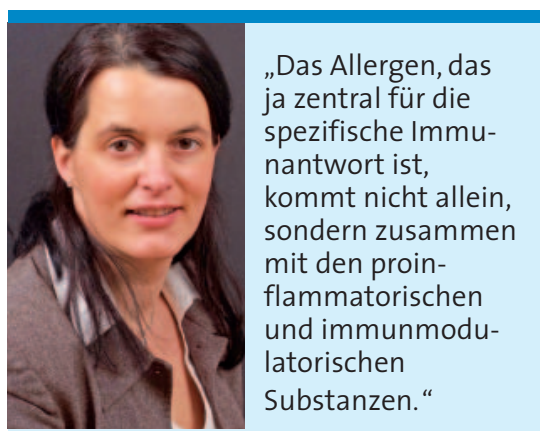

Prof. Dr. Claudia Traidl-Hoffmann ZAUM - Zentrum Allergie und Umwelt der TU München
? Ihre Arbeitsgruppe konnte nachweisen, dass Pollen im feuchten Milieu nicht nur Allergene, sondern auch PALMs - pollenassoziierte Lipidmediatoren - freisetzen. Um was für Lipide handelt es sich hierbei?

Traidl-Hoffmann: Es sind Prostaglandinoder Leukotrien-ähnliche Substanzen, die Abbauprodukte der Linol- und der Linolensäure sind und sowohl eine proinflammatorische als auch eine immunmodulatorische Wirkung haben. Diese Lipide werden zum Teil bereits in den Pollen, zum Teil aber auch noch nach ihrer Freisetzung durch Autooxidation gebildet. Zudem tragen wohl auch enzymgesteuerte Mechanismen dazu bei, dass sie umgewandelt werden.Beim proinflammatorischen Effekt wissen wir inzwischen aus humanen Zellkulturversuchen, dass er über Leukotrien-B4-Rezeptoren vermittelt wird und sowohl eine Chemotaxis als auch eine Degranulation von neutrophilen und eosinophilen Granulozyten auslöst, sodass lokal Zytokine freigesetzt werden. Dieser Effekt ist völlig unabhängig von einer Sensibilisierung - er kann also bei Allergikern und Nicht-Allergikern auftreten.

Der immunmodulatorische Effekt wird vermutlich über zwei Wege vermittelt - zum einen über extrazelluläre Prostglandinrezeptoren und zum anderen über intrazelluläre PPAR-gamma-Rezeptoren (peroxisome proliferator activator receptor gamma; Anm. der Redaktion), die sich auf oder in humanen dendritischen Zellen befinden. Beides führt letztendlich dazu, dass die Produktion von IL-12, dem zentralen Interleukin der Th1-In- duktion, blockiert wird. Dadurch kommt es zu einer proallergischen Verschiebung der Immunantwort in Richtung Th2. Das haben wir im Zellversuch gezeigt und konnten es im Mausmodell bestätigen.

? Welche Folgen könnten diese Effekte im Körper am Ort der Pollenexposition haben? Traidl-Hoffmann: Wir gehen davon aus, das die PALMs beim Auslösen einer Allergie einen adjuvanten Effekt haben: Das Allergen, das ja zentral für die spezifische Immunantwort ist, kommt nicht allein, sondern zusammen mit den proinflammatorischen und immunmodulatorischen Substanzen. Diese sind dann eventuell das Zünglein an der Waage und führen letztendlich erst dazu, dass sich überhaupt eine allergische Reaktion entwickelt.

? Wie Sie sagen, treten die proinflammatorischen Effekte nicht nur bei Allergikern, sondern auch bei Menschen ohne Allergien auf. Welche neuen Ansätze ergeben sich hieraus für die Allergologie?

Traidl-Hoffmann: Das wirft die Frage auf ob Pollen wirklich nur Allergien, oder bei Menschen ohne Sensibilisierung einfach auch entzündliche Reaktionen hervorrufen. Demnach müssen wir bei den Pollen vielleicht ein wenig über den Tellerrand der Allergie hinausschauen. So gibt es Daten, nach denen kardiovaskuläre und pulmonale Erkrankungen mit dem Pollenflug korrelieren. Auch unspezifische Augenreizungen, die gehäuft während der Pollensaison auf- treten, sind nicht auf eine Allergie, sondern womöglich auf die proinflammatorischen Effekte der PALMs zurückzuführen.

? Liefern Ihre Untersuchungen auch eine Erklärung dafür, warum Pollen in Gebieten mit hoher Luftschadstoffbelastung ein besonders hohes Allergenpotential haben?

Traidl-Hoffmann: Ja, wir haben Pollen aus verkehrsbelasteten und ländlichen Gebieten analysiert und verglichen. Dabei zeigte sich, dass die Pollen aus den verkehrsbelasteten Gebieten verstärkt proinflammatorische Substanzen freisetzen. Das passt in das Bild, dass in der Stadt vermehrt Allergien auftreten. Ich möchte zwar nicht sagen, dass die PALMs für alles verantwortlich sind, aber sie können durchaus ein Teil in dem Puzzle sein, warum Allergien in Städten häufiger vorkommen.

? Könnten sich aus Ihren Erkenntnissen neue Ansätze für die Prävention oder Therapie von Pollen-Allergien ergeben?

Traidl-Hoffmann: Das ist unser Ziel. Wenn wir die involvierten Rezeptoren identifizieren, könnten wir hieraus neue Therapieansätze entwickeln. Und wenn wir wissen, welche Luftschadstoffe zur Hochregulierung der proinflammatorischen Substanzen in den Pollen führen, könnten wir versuchen, präventiv vorzugehen und diese Luftschadstoffe gezielt zu reduzieren.

Das Interview führte Petra Eiden, Berlin 